Publ. RIMS, Kyoto Univ.

41 (2005), 397-416

\title{
Projected Canonical Curves and the Clifford Index
}

\author{
Dedicated to Professor Makoto Namba on his 60th birthday \\ By
}

Kazuhiro KonNo*

\section{Introduction}

We shall work over the complex number field $\mathbb{C}$. Let $X$ be a non-singular projective curve of genus $g$. We always assume that it is non-hyperelliptic and sometimes identify it with its canonical image in $\mathbb{P} H^{0}\left(X, K_{X}\right)$. By Max Noether's theorem, the canonical ring of $X$ is generated in degree one and the canonical map is a projectively normal embedding. Furthermore, a well-known theorem of Enriques-Petri states that $X$ is cut out by hyperquadrics if $\operatorname{Cliff}(X)$, the Clifford index of $X$, is bigger than one. Then Mark Green [4] conjectured that the non-vanishing of a certain higher syzygy can be characterized by the Clifford index, which has been verified in many cases. From these, we learn that $\operatorname{Cliff}(X)$ reflects the algebraic structure on $X$ better than the gonality $\operatorname{gon}(X)$, while two invariants are almost equivalent [2].

For a non-negative integer $k$, we denote by $X^{(k)}$ the $k$-th symmetric product of $X$ whose points are considered as effective divisors of degree $k$ on $X$. When $k=0$, we understand that $X^{(0)}$ is one point corresponding to the zero divisor. Let $\mathcal{D}_{k}$ be the open subset of $X^{(k)}$ consisting of effective divisors $D$ which spans scheme theoretically a $(k-1)$-plane $\langle D\rangle$ in $\mathbb{P} H^{0}\left(X, K_{X}\right)$. For $D \in \mathcal{D}_{k}$, we put $K_{X, D}=K_{X}-[D]$, where $[D]$ denotes the line bundle associated to $D$. We let $\Phi_{K_{X, D}}: X \rightarrow \mathbb{P} H^{0}\left(X, K_{X, D}\right)$ be the rational map associated to the complete linear system $\left|K_{X, D}\right|$.

In this article, we consider two kinds of uniformity questions with respect to $\mathcal{D}_{k}$ :

Communicated by S. Mukai. Received October 24, 2003.

2000 Mathematics Subject Classification(s): 14 H51.

* Department of Mathematics, Graduate School of Science, Osaka University, Toyonaka, Osaka 560-0043, Japan.

e-mail: konno@math.wani.osaka-u.ac.jp

(C) 2005 Research Institute for Mathematical Sciences, Kyoto University. All rights reserved. 
- When is $K_{X, D}$ normally generated for any $D \in \mathcal{D}_{k}$ ?

- When is the image $\Phi_{K_{X, D}}(X)$ cut out by hyperquadrics for any $D \in \mathcal{D}_{k}$ ?

In view of Max Noether theorem and Enriques-Petri theorem, we have a hope that each of the algebraic questions can be answered in terms of the Clifford index. We remark that the analogous geometric uniformity property about the base point freeness or the very ampleness of $K_{X, D}$ can be easily characterized by the gonality (see $\S 1$ ).

Now, our main result is the following:

Main Theorem. Let $X$ be a canonical curve of genus $g$ and $k$ an integer with $0 \leq k<g-1$.

(1) Assume that $k \leq 33$. Then $K_{X, D}$ is normally generated for any $D \in \mathcal{D}_{k}$ if and only if $\operatorname{Cliff}(X) \geq k+1$.

(2) Assume that $k \leq 5$. Then $\Phi_{K_{X, D}}(X)$ is cut out by quadrics for any $D \in \mathcal{D}_{k}$ if and only if $\operatorname{Cliff}(X) \geq k+2$.

In each of (1) and (2), the "if" part is an easy application of remarkable theorems due to Green-Lazarsfeld [5] and Lange-Sernesi [7], respectively. So the difficulty is in finding a special configuration of $k$ points which breaks the ideal property. We remark that the unpleasant restrictions on $k$ are closely related to the conjecture in $[3$, p.175] on curves of high Clifford dimension, which is known so far to hold when the Clifford index is at most 33 (see, [3]). Indeed, we can remove the restriction on $k$ in (1) if the conjecture is true. We also remark that (2) for $k=1$ is an unpublished result of Mukai.

The plan of the paper is as follows. In $\S 1$, we collect known results about the Clifford index from [3] and [2]. We also consider the geometric analogue of our questions and give an answer in Proposition 1.1. In $\S 2$, we show Theorems 2.1 and 2.2 which together with [5] and [7] give us Main Theorem. In $\S 3$, we describe the quadric hull of curves with $\operatorname{Cliff}(X)=2$ to see what happens when we consider the projection of $X$ from a point $x \in X$, and show Theorem 3.1.

\section{$\S 1$. Clifford Index}

Let $X$ be a non-singular projective curve of genus $g \geq 2$. The gonality of $X$, which we denote by $\operatorname{gon}(X)$, is by definition the minimum of the degrees of surjective morphisms of $X$ to $\mathbb{P}^{1}$. It is known that $\operatorname{gon}(X) \leq[(g+3) / 2]$, where $[x]$ denotes the integer part of a real number $x$. 
We have another invariant, the Clifford index, introduced by H. Martens. Let us recall the definition and some related topics. See [3] and [2] for the detail. The Clifford index of a line bundle $L \in \operatorname{Pic}(X)$ is

$$
\operatorname{Cliff}(L):=\operatorname{deg} L-2 h^{0}(X, L)+2
$$

Note that the Riemann-Roch theorem gives us

$$
h^{0}(X, L)+h^{1}(X, L)=g+1-\operatorname{Cliff}(L)
$$

The Clifford index of $X$ is

$$
\operatorname{Cliff}(X):=\min \left\{\operatorname{Cliff}(L) \mid h^{0}(X, L)>1, h^{1}(X, L)>1\right\}
$$

for $g \geq 4$. When $g=2$, we put $\operatorname{Cliff}(X)=0$. When $g=3$, we put $\operatorname{Cliff}(X)=0$ or 1 according to whether $X$ is hyperelliptic or not. A line bundle as in the right hand side of the definition of $\operatorname{Cliff}(X)$ is said to contribute to the Clifford index and, among them, those $L$ with $\operatorname{Cliff}(L)=\operatorname{Cliff}(X)$ are said to compute the Clifford index.

There is a striking relation between the gonality and the Clifford index. In fact, it is shown in [2] that $\operatorname{Cliff}(X)=\operatorname{gon}(X)-2$ or $\operatorname{gon}(X)-3$ and that the latter is a special case. This says in particular that $\operatorname{Cliff}(X)$ is not necessarily computed by a pencil. So we need another invariant especially for curves with $\operatorname{Cliff}(X)=\operatorname{gon}(X)-3$. We put

$$
r(X):=\min \left\{h^{0}(X, L)-1 \mid L \text { computes } \operatorname{Cliff}(X)\right\}
$$

and call it the Clifford dimension of $X$ following [3]. A line bundle $L$ computing $\operatorname{Cliff}(X)$ and $h^{0}(X, L)=r(X)+1$ is said to compute the Clifford dimension. Recall that a non-singular plane curve of degree $d \geq 5$ has $\operatorname{gon}(X)=d-1$, $\operatorname{Cliff}(X)=d-4$ and $r(X)=2$ (see, e.g. [8]). In this case, a line on $\mathbb{P}^{2}$ induces on $X$ a line bundle computing the Clifford dimension. When $r(X) \geq 3$, it is shown in [3] that, for $L$ computing the Clifford dimension, we have either (i) $\operatorname{deg} L=4 r(X)-3, K_{X}=2 L$, or (ii) $\operatorname{deg} L \geq 6 r(X)-6, g \geq 8 r(X)-7$. It is conjectured in [3] that the latter case cannot happen, which is true when $3 \leq r(X) \leq 9$. We call $(X, L)$ is an ELMS curve if $\operatorname{deg} L=4 r(X)-3$ holds for $L$ computing the Clifford dimension $r(X) \geq 3$.

For a line bundle $L$ on $X$, we denote by $\Phi_{L}: X \rightarrow \mathbb{P} H^{0}(X, L)$ the rational map associated with $|L|$. We denote by $\operatorname{Quad}(X, L)$ the intersection of all hyperquadrics through $\Phi_{L}(X)$ and call it the quadric hull of $\Phi_{L}(X)$. Here we recall the following beautiful theorem due to Green-Lazarsfeld [5] and LangeSernesi [7], which will play an important role in the proof of Main Theorem. 
Theorem 1.1 ([5] and [7]). Let $L$ be a very ample line bundle on a nonsingular projective curve $X$.

(1) If $\operatorname{Cliff}(L) \leq \operatorname{Cliff}(X)-1$, then $L$ is normally generated. That is, the multiplication map $\operatorname{Sym}^{n} H^{0}(X, L) \rightarrow H^{0}(X, n L)$ is surjective for $\forall n \in \mathbb{N}$.

(2) If $\operatorname{Cliff}(L) \leq \operatorname{Cliff}(X)-2$, then

$$
\operatorname{Quad}(X, L)=\Phi_{L}(X) \bigcup\left(\text { trisecant lines to } \Phi_{L}(X)\right)
$$

Lemma 1.1. Let $L$ be a special line bundle on $X$. Assume that there is a positive integer $\ell$ such that $\operatorname{deg} L \geq 2 g-1+\ell-\operatorname{gon}(X)$ holds. Then for any effective divisor $Z$ of degree $\ell$, the restriction map $H^{0}(X, L) \rightarrow H^{0}\left(Z,\left.L\right|_{Z}\right)$ is surjective.

Proof. We assume that the restriction map is not surjective and show that this leads us to a contradiction. Since we have assumed that $h^{0}(X, L-Z) \geq$ $h^{0}(X, L)-\ell+1$ and $h^{1}(X, L) \neq 0$, we have

$$
\begin{aligned}
h^{0}\left(X, K_{X}-L+Z\right) & =\operatorname{deg}\left(K_{X}-L+Z\right)+1-g+h^{0}(X, L-Z) \\
& =-h^{0}(X, L)+h^{1}(X, L)+\ell+h^{0}(X, L-Z) \\
& \geq 2 .
\end{aligned}
$$

On the other hand, since $\operatorname{deg} L \geq 2 g-1+\ell-\operatorname{gon}(X)$, we get

$$
\operatorname{deg}\left(K_{X}-L+Z\right)=2 g-2-\operatorname{deg} L+\ell<\operatorname{gon}(X) .
$$

This is absurd.

Let $k$ be an integer with $0 \leq k \leq g$. We denote by $\mathcal{D}_{k}$ the set of all effective divisors $D$ of degree $k$ with $h^{0}\left(X, K_{X}\right)-h^{0}\left(X, K_{X}-[D]\right)=k$. That is, an element $D \in \mathcal{D}_{k}$ spans scheme theoretically a $(k-1)$ plane in $\mathbb{P} H^{0}\left(X, K_{X}\right)$. For $D \in X^{(k)}$, we put $K_{X, D}:=K_{X}-[D]$. We have $h^{0}(X,[D])=k+1-g+$ $h^{0}\left(X, K_{X, D}\right)$ by the Riemann-Roch theorem and the duality theorem. Hence, for $D \in X^{(k)}$, we have $D \in \mathcal{D}_{k}$ if and only if $h^{0}(X,[D])=1$.

We consider the geometric analogue of our questions in Introduction.

Proposition 1.1. Let $k$ and $\ell$ be non-negative integers with $0 \leq k+\ell \leq$ g. Then the following hold.

(1) $\mathcal{D}_{k}=X^{(k)}$ holds if and only if $\operatorname{gon}(X) \geq k+1$. 
(2) Assume that $\ell>0$ and consider the map $\Psi: \mathcal{D}_{k} \times X^{(\ell)} \rightarrow X^{(k+\ell)}$ defined by $\Psi((D, Z))=D+Z$. The image of $\Psi$ is contained in $\mathcal{D}_{k+\ell}$ if and only if $\operatorname{gon}(X) \geq k+\ell+1$. In particular,

(a) $K_{X, D}$ is free from base points for $\forall D \in \mathcal{D}_{k}$ if and only if $\operatorname{gon}(X) \geq$ $k+2$

(b) $K_{X, D}$ is very ample for $\forall D \in \mathcal{D}_{k}$ if and only if $\operatorname{gon}(X) \geq k+3$

(c) $\Phi_{K_{X, D}}(X)$ has no trisecant lines for $\forall D \in \mathcal{D}_{k}$ if and only if $\operatorname{gon}(X) \geq$ $k+4$

Proof. (1) directly follows from the definition of gonality. (2) If $\operatorname{gon}(X)>$ $k+\ell$, then we have $\operatorname{Im}(\Psi) \subset X^{(k+\ell)}=\mathcal{D}_{k+\ell}$ by (1). Suppose that $\operatorname{gon}(X)=$ $d \leq k+\ell$. Let $E \in g_{d}^{1}$ be a general member. Assume that $k<d$. We add general $k+\ell-d$ points to $E$ to get a divisor $G \in X^{(k+\ell)} \backslash \mathcal{D}_{k+\ell}$. If we take any subdivisor $D$ of degree $k$ from $G$, then we have $D \in \mathcal{D}_{k}$ and $Z=G-D \in X^{(\ell)}$. Assume that $k \geq d$. We decompose $E$ as $E=E_{1}+E_{2}$ with $\operatorname{deg} E_{1}=d-1$ and $\operatorname{deg} E_{2}=1$. We add general $k-d+1$ points to $E_{1}$ to get a divisor $D \in \mathcal{D}_{k}$. Similarly, we add any $\ell-1$ points to $E_{2}$ to get $Z \in X^{(\ell)}$. Then $D+Z \notin \mathcal{D}_{k+\ell}$, because $E$ is a subdivisor of $D+Z$.

Now, let $D \in \mathcal{D}_{k}$ and consider the restriction map

$$
H^{0}\left(X, K_{X, D}\right) \rightarrow H^{0}\left(Z,\left.K_{X, D}\right|_{Z}\right)
$$

for any $Z \in X^{(\ell)}$. Since $D \in \mathcal{D}_{k}$, we have $h^{1}\left(X, K_{X, D}\right)=1$. Hence the restriction map is surjective if and only if $h^{1}\left(X, K_{X, D}-Z\right)=1$, that is, $D+Z \in$ $\mathcal{D}_{k+\ell}$. So, by putting $\ell=1,2,3$, we respectively get (a), (b), (c). We remark that the "if part" also follows from Lemma 1.1 applied for $L=K_{X, D}$.

\section{§2. Proof of Main Theorem}

In this section, we shall show Main Theorem in Introduction (and more) with several lemmas. The following shows its "if" parts.

Lemma 2.1. Let $k$ be a non-negative integer with $0 \leq k<g-1$. Then the following hold.

(1) $K_{X, D}$ is normally generated for any $D \in \mathcal{D}_{k}$ if $\operatorname{Cliff}(X) \geq k+1$.

(2) $\operatorname{Quad}\left(X, K_{X, D}\right) \simeq X$ for any $D \in \mathcal{D}_{k}$ if $\operatorname{Cliff}(X) \geq k+2$. 
Proof. Recall that $\operatorname{gon}(X)-3 \leq \operatorname{Cliff}(X) \leq \operatorname{gon}(X)-2$. Then it follows from Lemma 1.1 applied for $\ell=2,3$ that $K_{X, D}$ is very ample if $\operatorname{Cliff}(X) \geq k+1$, and that $\Phi_{K_{X, D}}(X)$ has no trisecant lines if $\operatorname{Cliff}(X) \geq k+2$. Note that we have $\operatorname{Cliff}\left(K_{X, D}\right)=k$ for $D \in \mathcal{D}_{k}$. Therefore, Theorem 1.1 gives us the assertion.

We look for a special configuration of $k$ points which prevents $K_{X, D}$ from being normally generated or, $\Phi_{K_{X, D}}(X)$ from being cut out by hyperquadrics.

\section{§2.1. Plane curves}

Let $X \subset \mathbb{P}^{2}$ be a non-singular plane curve of degree $d \geq 5$. We take a line $\ell \subset \mathbb{P}^{2}$ such that $X \cap \ell$ consists of $d$ distinct points. Let $k$ be a positive integer with $0<k \leq d-4$. We take $k$ points $x_{1}, \ldots, x_{k}$ from $X \cap \ell$ and put $D=x_{1}+\cdots+x_{k}$. Then $D \in \mathcal{D}_{k}$. Let $\mu: W \rightarrow \mathbb{P}^{2}$ be the blow-up with center $\cup_{i=1}^{k} x_{i}$, and put $E=\sum_{i=1}^{k} \mu^{-1}\left(x_{i}\right)$. We identify $X$ with its proper transform in $W$. Then $X$ is linearly equivalent to $d \mu^{*} \ell-E$ on $W$ and $K_{X, D}$ is induced by $(d-3) \mu^{*} \ell-E$. It follows from the cohomology long exact sequence for

$$
0 \rightarrow \mathcal{O}_{W}\left(-3 \mu^{*} \ell\right) \rightarrow \mathcal{O}_{W}\left((d-3) \mu^{*} \ell-E\right) \rightarrow \mathcal{O}_{X}\left(K_{X, D}\right) \rightarrow 0,
$$

we have $H^{0}\left(W,\left[(d-3) \mu^{*} \ell-E\right]\right) \simeq H^{0}\left(X, K_{X, D}\right)$.

Lemma 2.2. Let $X$ be a non-singular plane curve of degree $k+4$. Then there exists a divisor $D \in \mathcal{D}_{k}$ such that $K_{X, D}$ is not normally generated.

Proof. Since the restriction map $H^{0}\left(W,(k+1) \mu^{*} \ell-E\right) \rightarrow H^{0}\left(X, K_{X, D}\right)$ is an isomorphism, we see that the multiplication map $\operatorname{Sym}^{2} H^{0}\left(X, K_{X, D}\right) \rightarrow$ $H^{0}\left(X, 2 K_{X, D}\right)$ factors through $H^{0}\left(W, 2(k+1) \mu^{*} \ell-2 E\right)$. Consider the cohomology long exact sequence for

$$
0 \rightarrow \mathcal{O}_{W}\left((k-2) \mu^{*} \ell-E\right) \rightarrow \mathcal{O}_{W}\left(2(k+1) \mu^{*} \ell-2 E\right) \rightarrow \mathcal{O}_{X}\left(2 K_{X, D}\right) \rightarrow 0 .
$$

We shall show that $H^{1}\left(W, 2(k+1) \mu^{*} \ell-2 E\right)=0$ and $H^{1}\left(W,(k-2) \mu^{*} \ell-E\right) \simeq \mathbb{C}$ to show that the restriction map $H^{0}\left(W, 2(k+1) \mu^{*} \ell-2 E\right) \rightarrow H^{0}\left(X, 2 K_{X, D}\right)$ is not surjective.

For this purpose, let $\tilde{\ell}$ be the proper transform of $\ell$ by $\mu$. Consider the cohomology long exact sequence for

$$
0 \rightarrow \mathcal{O}_{W}\left((k-3) \mu^{*} \ell\right) \rightarrow \mathcal{O}_{W}\left((k-2) \mu^{*} \ell-E\right) \rightarrow \mathcal{O}_{\tilde{\ell}}(-2) \rightarrow 0 .
$$

Since $H^{i}\left(W,(k-3) \mu^{*} \ell\right) \simeq H^{i}\left(\mathbb{P}^{2}, \mathcal{O}(k-3)\right)$, we see that it vanishes for $i=1,2$. Hence $H^{1}\left(W,(k-2) \mu^{*} \ell-E\right) \simeq H^{1}(\tilde{\ell}, \mathcal{O}(-2)) \simeq \mathbb{C}$. We next show the vanishing 
of $H^{1}\left(W, 2(k+1) \mu^{*} \ell-2 E\right)$. We consider the cohomology long exact sequences for

$$
0 \rightarrow \mathcal{O}_{W}\left(2 k \mu^{*} \ell\right) \rightarrow \mathcal{O}_{W}\left((2 k+1) \mu^{*} \ell-E\right) \rightarrow \mathcal{O}_{\tilde{\ell}}(k+1) \rightarrow 0
$$

and

$$
0 \rightarrow \mathcal{O}_{W}\left((2 k+1) \mu^{*} \ell-E\right) \rightarrow \mathcal{O}_{W}\left(2(k+1) \mu^{*} \ell-2 E\right) \rightarrow \mathcal{O}_{\tilde{\ell}}(2) \rightarrow 0 .
$$

We get from the first one that $H^{1}\left(W,(2 k+1) \mu^{*} \ell-E\right)=0$. Then the second one shows that $H^{1}\left(W, 2(k+1) \mu^{*} \ell-2 E\right)$ also vanishes. Therefore, $K_{X, D}$ is not normally generated.

Lemma 2.3. Let $X$ be a non-singular plane curve of degree $k+5$. Then there exists a divisor $D \in \mathcal{D}_{k}$ such that $\operatorname{Quad}\left(X, K_{X, D}\right)$ contains a conic curve.

Proof. Since $H^{0}\left(W,(k+2) \mu^{*} \ell-E\right) \simeq H^{0}\left(X, K_{X, D}\right), \Phi_{K_{X, D}}(X)$ is contained in the surface $V$ obtained as the $(k+2)$-th Veronese embedding of $\mathbb{P}^{2}$ followed by the linear projection from $\langle D\rangle$. Consider now the cohomology long exact sequence for

$$
0 \rightarrow \mathcal{O}_{W}\left((k-1) \mu^{*} \ell-E\right) \rightarrow \mathcal{O}_{W}\left((2 k+4) \mu^{*} \ell-2 E\right) \rightarrow \mathcal{O}_{X}\left(2 K_{X, D}\right) \rightarrow 0 .
$$

If $k=1$, then we have $H^{0}\left(W, 6 \mu^{*} \ell-2 E\right) \simeq H^{0}\left(X, 2 K_{X, D}\right)$. We see that the quadric hull of $\Phi_{K_{X, D}}(X)$ is $V$ which is isomorphic to the Hirzebruch surface of degree 1. If $k>1$, then $H^{0}\left(W,(k-1) \mu^{*} \ell-E\right) \neq 0$. It follows that there are more quadrics through $X$ than $V$. Let $\tilde{\ell}$ be the proper transform of $\ell$ by $\mu$. Then $\left((k-1) \mu^{*} \ell-E\right) \cdot \tilde{\ell}=-1$ and it follows that $\tilde{\ell}$ is a fixed component of $\left|(k-1) \mu^{*} \ell-E\right|$. From this, we see that the quadric hull of $X$ contains $\tilde{\ell}$. Note that $\tilde{\ell}$ is of degree 2 in $\mathbb{P} H^{0}\left(X, K_{X, D}\right)$.

\section{§2.2. $\quad$ ELMS curves}

We let $(X, L)$ be an ELMS curve. We put $r=h^{0}(X, L)-1 \geq 3$. Then $\operatorname{deg} L=4 r-3$ and $\operatorname{Cliff}(X)=2 r-3$. Since $K_{X}=2 L$, we have $g=4 r-2$. It is known that $\Phi_{L}$ is a projectively normal embedding and $\Phi_{L}(X)$ has no $(2 s+2)$-secant $s$-planes for $1 \leq s \leq r-2$ (see, [3, Lemma 1.1]). It is shown in [3, Theorem 3.7] that there is a $(2 r-3)$ secant $r-2$ space divisor $Z$ with respect to $|L|$. Then $Z \in \mathcal{D}_{2 r-3}$ and $|L-Z|$ is a pencil of minimal degree $2 r$.

Lemma 2.4. Let $(X, L)$ be an ELMS curve of Clifford dimension $r$. Then there exists a divisor $D \in \mathcal{D}_{2 r-3}$ such that $K_{X, D}$ fails to be normally generated. 
Proof. We put $D=Z$. By the free-pencil-trick, we have an exact sequence

$$
0 \rightarrow H^{0}(X, D) \rightarrow H^{0}(X, L-D) \otimes H^{0}(X, L) \rightarrow H^{0}\left(X, K_{X, D}\right)
$$

On one hand, we have $\operatorname{rank}\left(H^{0}(X, L-D) \otimes H^{0}(X, L) \rightarrow H^{0}\left(X, K_{X, D}\right)\right)=$ $2 r+1$, because we have $h^{0}(X, L)=r+1$ and $h^{0}(X, D)=1$. On the other hand, we have $h^{0}\left(X, K_{X, D}\right)=g-(2 r-3)=2 r+1$. Hence the multiplication map $H^{0}(X, L-D) \otimes H^{0}(X, L) \rightarrow H^{0}\left(X, K_{X, D}\right)$ is surjective.

We shall show that $H^{0}\left(X, K_{X, D}\right) \otimes H^{0}\left(X, K_{X, D}\right) \rightarrow H^{0}\left(X, 2 K_{X, D}\right)$ is not surjective. By what we have seen above, it suffices to show that $H^{0}(X, L-$ $D) \otimes H^{0}(X, L) \otimes H^{0}\left(X, K_{X, D}\right) \rightarrow H^{0}\left(X, 2 K_{X, D}\right)$ is not surjective. Since it factors through $H^{0}(X, L-D) \otimes H^{0}\left(X, K_{X, D}+L\right) \rightarrow H^{0}\left(X, 2 K_{X, D}\right)$, we only have to show that this map is not surjective. Again by the free-pencil-trick, we have the exact sequence

$$
\begin{aligned}
0 \rightarrow H^{0}\left(X, K_{X}\right) & \rightarrow H^{0}(X, L-D) \otimes H^{0}\left(X, K_{X, D}+L\right) \\
& \rightarrow H^{0}\left(X, 2 K_{X, D}\right) \rightarrow H^{1}\left(X, K_{X}\right) \rightarrow 0,
\end{aligned}
$$

because $2 L=K_{X}$. Since $h^{1}\left(X, K_{X}\right)=1$, we get the assertion.

In order to study the quadric hull, we first show a conditional result:

Lemma 2.5. Let $(X, L)$ be an ELMS curve and $\Phi_{L}: X \rightarrow \mathbb{P} H^{0}(X, L)$ the Clifford embedding. Put $r=h^{0}(X, L)-1$. Assume that $\Phi_{L}(X)$ has a $(2 r-3)$-secant $(r-2)$-plane $\Lambda$ such that there is a non-singular rational curve $C$ of degree $d$ in $\Lambda$ passing through $2 d+1$ points in $\Phi_{L}(X) \cap \Lambda, 1 \leq d \leq r-2$. Then there exists a divisor $D \in \mathcal{D}_{2 r-4}$ such that $\operatorname{Quad}\left(X, K_{X, D}\right)$ contains an extra rational curve.

Proof. We choose one point $P$ from $\Phi_{L}(X) \cap C$ and define a divisor $D \in$ $\mathcal{D}_{2 r-4}$ with $\Phi_{L}(X) \cap \Lambda \backslash\{P\}$. Since $L$ is projectively normal and $K_{X}=2 L$, any hyperquadrics through $\Phi_{K_{X, D}}(X)$ can be interpreted as a hyperquartic on $\mathbb{P} H^{0}(X, L)$ which passes through $\Phi_{L}(X) \cap \Lambda$ and vanishes twice on $D$. Since $C \subset \Lambda$ is a non-singular rational curve of degree $d$ through $2 d+1$ points from $\Phi_{L}(X) \cap \Lambda$ and containing $2 d$ points in $D$, such a quartic must contain $C$ as well for a simple degree reason: $4 d-(2 \times 2 d+1)=-1<0$.

Corollary 2.1. Let $(X, L)$ be an ELMS curve of Clifford dimension $r \leq 4$. Then there exists a divisor $D \in \mathcal{D}_{2 r-4}$ such that $\operatorname{Quad}\left(X, K_{X, D}\right)$ contains an extra rational curve. 
Proof. Let $\Lambda$ be a $(2 r-3)$-secant $(r-2)$-plane. If $r=3$, then $\Lambda$ is a trisecant line and we can apply Lemma 2.5 putting $C=\Lambda$. Assume that $r=4$. Then $\Lambda \simeq \mathbb{P}^{2}$ and $\Phi_{L}(X) \cap \Lambda$ consists of five points. Hence we can find a conic through all the five points. Recall that $\Phi_{L}(X)$ has no $(2 s+2)$-secant $s$-planes for $1 \leq s \leq r-2$. It follows that the conic cannot be a double line. If the conic is irreducible, then we are done by Lemma 2.5. If the conic is reducible, one component must be a line $C$ which contains 3 points from $\Phi_{L}(X) \cap \Lambda$. Hence, also in this case, we can apply Lemma 2.5.

\section{$\S 2.3$. Summary}

We summarize here the results obtained above in two theorems.

Theorem 2.1. $\quad$ Suppose that $\operatorname{Cliff}(X) \leq k$. Then there exists a divisor $D \in \mathcal{D}_{k}$ such that $K_{X, D}$ fails to be normally generated in the following cases:

(1) $\operatorname{gon}(X) \leq k+2$.

(2) $X$ is a non-singular plane curve of degree $k+4$.

(3) $X$ is an ELMS curve with $\operatorname{Cliff}(X)=k$.

Proof. Because a projectively normal line bundle is necessarily very ample, we have (1) by Proposition 1.1, (b). Hence we can assume that $\operatorname{gon}(X)=$ $k+3$ and $\operatorname{Cliff}(X)=k$. Then (2) and (3) are covered by Lemmas 2.2 and 2.4, respectively.

Theorem 2.2. $\quad$ There exists a divisor $D \in \mathcal{D}_{k}$ such that $\operatorname{Quad}\left(X, K_{X, D}\right)$ contains an extra variety if $X$ satisfies either

(1) $\operatorname{gon}(X) \leq k+3$, or

(2) $\operatorname{Cliff}(X)=k+1$ and $r(X) \leq 4$.

Proof. (2) follows from Lemma 2.3 and Corollary 2.1. (1): Assume that $\operatorname{gon}(X) \leq k+3$. Then it follows from (c) of Proposition 1.1 that $\Phi_{K_{X, D}}(X)$ has a trisecant line for some $D \in \mathcal{D}_{k}$. It is then clear that $\operatorname{Quad}\left(X, K_{X, D}\right)$ contains such a trisecant line.

Now, the rest of Main Theorem follows from Theorems 2.1 and 2.2, if we note that the conjecture of Eisenbud-Lange-Martens-Schreyer is true when the Clifford dimension is less than ten. Q.E.D. of Main Theorem 
For $D \in \mathcal{D}_{k}$, we let $\operatorname{Quad}\left(X, K_{X},\langle D\rangle\right)$ be the intersection of all hyperquadrics through $X \subset \mathbb{P} H^{0}\left(X, K_{X}\right)$ which are singular along $\langle D\rangle$. We call it the singular quadric hull of $X$ with ridge $\langle D\rangle$. Note that such a hyperquadric through $X$ is in one-to-one correspondence with a hyperquadric through $\Phi_{K_{X, D}}(X) \subset \mathbb{P} H^{0}\left(X, K_{X, D}\right)$. Hence, we get:

Corollary 2.2. $\quad$ Let $X \subset \mathbb{P} H^{0}\left(X, K_{X}\right)$ be a canonical curve and $k$ an integer with $0 \leq k \leq 5$. Then

$$
\operatorname{Quad}\left(X, K_{X},\langle D\rangle\right)=X *\langle D\rangle
$$

holds for any $D \in \mathcal{D}_{k}$ if and only if $\operatorname{Cliff}(X) \geq k+2$, where the operation * denotes the projective join (see $[6, \S 4])$. In other words, $X$ can be recovered from the singular quadric hull as its base if and only if $\operatorname{Cliff}(X) \geq k+2$.

We close the section by showing how to find a trisecant line geometrically when $\operatorname{gon}(X)=k+3$.

Lemma 2.6. Let $X$ be a non-singular curve of $\operatorname{gon}(X)=k+3$. Then there exists a divisor $D \in \mathcal{D}_{k}$ such that $\operatorname{Quad}\left(X, K_{X, D}\right)$ contains a trisecant line to $\Phi_{K_{X, D}}(X)$.

Proof. We denote by $f: X \rightarrow \mathbb{P}^{1}$ the surjective morphism corresponding to a $g_{k+3}^{1}$. Let $F$ be a general fiber of $f$. We can assume that $F$ consists of $k+3$ distinct points. We choose $k$ points from $F$ to get a subdivisor $D$ of degree $k$. Then $D \in \mathcal{D}_{k}$. Let $\mathcal{E}$ be the saturated subsheaf of $f_{*} \omega_{X}$ generated by $H^{0}\left(\mathbb{P}^{1}, f_{*} \omega_{X}\right)$. Then it is of rank $k+2$, and the natural sheaf homomorphism $f^{*} \mathcal{E} \hookrightarrow f^{*} f_{*} \omega_{X} \rightarrow \omega_{X}$ induces a morphism $\psi: X \rightarrow \mathbb{P}(\mathcal{E})$. We remark that $\psi$ followed by the morphism defined by the tautological line bundle $H$ on $\mathbb{P}(\mathcal{E})$ is nothing but the canonical map of $X$. Let $\Gamma$ be the fiber of $\mathbb{P}(\mathcal{E}) \rightarrow \mathbb{P}^{1}$ containing $F$. Then $\langle D\rangle$ is a linear subspace of $\Gamma \simeq \mathbb{P}^{k+1}$. We perform the elementary transformation along $\langle D\rangle$, that is, blow up $\mathbb{P}(\mathcal{E})$ with center $\langle D\rangle$ and then blow down the proper transform of $\Gamma$. Let $\mu: W \rightarrow \mathbb{P}(\mathcal{E})$ be the blowing-up with center $\langle D\rangle$ and $E$ the exceptional divisor. Then the morphism $\Phi$ of $W$ defined by $\left|\mu^{*} H-[E]\right|$ restricts to $X$ to give the morphism $\Phi_{K_{X, D}}$ and it factors through the elementary transformation. Let $\bar{\Gamma}$ be the proper transform of $\Gamma$ by $\mu$. Via the blow-down, $\bar{\Gamma}$ contracts to a line $\ell$ and we see that the three points in $F-D$ are mapped to $\ell$. In this way, we can find a trisecant line $\ell$ to $X \subset \mathbb{P} H^{0}\left(X, K_{X, D}\right)$. 


\section{§3. Quadric Hull of a Projected Canonical Curve}

In this section, we shall determine the quadric hull $\operatorname{Quad}\left(X, K_{X, D}\right)$ when $D \in \mathcal{D}_{1}$ and $\operatorname{Cliff}(X)=2$.

\section{§3.1. Special cases}

We first consider the easiest case that $g=5$ :

Lemma 3.1. If $X$ is a curve of genus five and $\operatorname{Cliff}(X)=2$, then $\operatorname{Quad}\left(X, K_{X, D}\right)=\mathbb{P} H^{0}\left(X, K_{X, D}\right)$ for any effective divisor $D$ of degree one. Furthermore $\Phi_{K_{X, D}}(X)$ is defined in $\mathbb{P} H^{0}\left(X, K_{X, D}\right)$ by

$$
\operatorname{rank}\left(\begin{array}{lll}
\ell_{1} & \ell_{2} & \ell_{3} \\
q_{1} & q_{2} & q_{3}
\end{array}\right)=1
$$

where the $\ell_{i}$ 's are linear forms and the $q_{j}$ 's are quadric forms in four variables.

Proof. Recall that $K_{X, D}$ induces a projectively normal embedding of $X$. We have $\operatorname{Sym}^{2} H^{0}\left(X, K_{X, D}\right) \simeq H^{0}\left(X, 2 K_{X, D}\right)$ by dimension count. Hence $\operatorname{Quad}\left(X, K_{X, D}\right)=\mathbb{P} H^{0}\left(X, K_{X, D}\right)$.

By the Hilbert-Burch theorem on the structure of Cohen-Macaulay ideal of codimension 2, we see that the minimal free resolution of the homogeneous ideal $I_{\Phi_{K_{X, D}}(X)}$ is given by

$$
0 \rightarrow R(-4) \oplus R(-5) \rightarrow R(-3)^{\oplus 3} \rightarrow I_{\Phi_{K_{X, D}(X)}} \rightarrow 0
$$

where $R=\mathbb{C}\left[Z_{0}, Z_{1}, Z_{2}, Z_{3}\right]$. Hence we get the assertion.

We assume that $g \geq 6$.

Lemma 3.2. If $X$ has several $g_{4}^{1}$ 's, then either $X$ is bi-elliptic, or $g \leq 9$. In the latter case, $X$ has either a simple $g_{6}^{2}$ or $X$ is of genus 9 with a simple $g_{8}^{3}$.

Proof. Two different $g_{4}^{1}$ 's give us a morphism $f: X \rightarrow \mathbb{P}^{1} \times \mathbb{P}^{1}$. We put $Y=f(X)$. We see that $Y$ is linearly equivalent to $4 \Delta$ or $2 \Delta$ according to whether $f$ is of degree one or two, where $\Delta$ is the diagonal divisor on $\mathbb{P}^{1} \times \mathbb{P}^{1}$. If $Y$ is linearly equivalent to $2 \Delta$, then its arithmetic genus is one. It follows that $Y$ is an elliptic curve, because if it were singular $X$ would have a $g_{2}^{1}$. Therefore, $X$ is a bi-elliptic curve in this case. Assume that $Y$ is linearly equivalent to $4 \Delta$. Then it is of arithmetic genus 9 and $|\Delta|$ induces on $X$ a $g_{8}^{3}$. If $Y$ is singular, the projection from the singular point gives $X$ a $g_{6}^{2}$. 
Remark 1. Martens-Mumford theorem (cf. [1]) states that, for $g \geq 6, X$ is a bi-elliptic curve if $X$ has a one dimensional family of $g_{4}^{1}$ 's.

Lemma 3.3. If $X$ is a bi-elliptic curve, then $\operatorname{Quad}\left(X, K_{X, D}\right)$ is a cone over an elliptic normal curve.

Proof. Let $f: X \rightarrow C$ be the double covering over an elliptic curve $C$. Because it is branched at $2 g-2$ distinct points on $C$, there is a line bundle $L^{\prime}$ of degree $g-1$ on $C$ such that $K_{X}=f^{*} L^{\prime}$. Since $K_{X, D}=K_{X}-[D]$, we can find a line bundle $L$ of degree $g-2$ on $C$ such that $\left|K_{X, D}-f^{*} L\right|$ contains an effective member. Now $\mathcal{E}:=f_{*} \mathcal{O}_{X}\left(K_{X, D}\right)$ is a locally free sheaf of rank 2 on $C$. We have $h^{0}(C, \mathcal{E})=h^{0}\left(X, K_{X, D}\right)=g-1$ and $h^{1}(C, \mathcal{E})=h^{1}\left(X, K_{X, D}\right)=1$. It follows that $\operatorname{deg}(\mathcal{E})=g-2$. Since $L$ is a subbundle of $\mathcal{E}$ of degree $g-2$, the quotient $\mathcal{E} / L$ is of degree zero. Since $h^{1}(C, \mathcal{E})=1$, we must have $\mathcal{E} / L \simeq \mathcal{O}_{C}$. Hence $\mathcal{E}=L \oplus \mathcal{O}_{C}$. The natural sheaf homomorphism $f^{*} \mathcal{E} \rightarrow \mathcal{O}_{X}\left(K_{X, D}\right)$ is surjective and induces a morphism $\psi: X \rightarrow \mathbb{P}_{C}(\mathcal{E})$ over $C$. We know that the map $\Phi_{K_{X, D}}$ is $\psi$ followed by a morphism of $\mathbb{P}_{C}(\mathcal{E})$ defined by the tautological line bundle. Therefore, $\Phi_{K_{X, D}}(X)$ is contained in the cone over a non-singular elliptic curve which is the image of the $\mathbb{P}^{1}$-bundle. Because the homogeneous ideal of the cone is generated by quadrics when $g \geq 6$, we see that it is nothing $\operatorname{but} \operatorname{Quad}\left(X, K_{X, D}\right)$.

Lemma 3.4. Assume that $X$ has a simple $g_{6}^{2}$. Then $\operatorname{Quad}\left(X, K_{X, D}\right)$ is a weak Del Pezzo surface of degree $g-2$.

Proof. We have $6 \leq g \leq 10$. The $g_{6}^{2}$ gives us a plane curve model $Y$ of $X$ such that $Y$ is a sextic with at most double points as its singularities. Note that the cardinality of the singular points is $10-g$ counting infinitely near ones. We blow $\mathbb{P}^{2}$ up at these double points $x_{1}, \cdots, x_{10-g}$ to get a rational surface $W$. Then $X \subset W$. We denote by $E_{i}$ the inverse image of $x_{i}$ on $W$. If $H$ denotes the pull-back to $W$ of a line on $\mathbb{P}^{2}$, then $X$ is linearly equivalent to $6 H-2 E_{1}-\cdots-2 E_{10-g}$. Let $\mu: \tilde{W} \rightarrow W$ be the blowing-up at $D \in X$. Put $E_{0}=\mu^{-1} D$. We denote the proper transform of $X$ in $\tilde{W}$ by the same symbol. Then $K_{X, D}$ is induced by $\tilde{L}=\left[3 \mu^{*} H-E_{0}-E_{1}-\cdots-E_{10-g}\right]$ on $\tilde{W}$. From the exact cohomology sequence for

$$
0 \rightarrow \mathcal{O}_{\tilde{W}}\left(-E_{0}\right) \rightarrow \mathcal{O}_{\tilde{W}}(2 \tilde{L}) \rightarrow \mathcal{O}_{X}\left(2 K_{X, D}\right) \rightarrow 0
$$

we see that $H^{0}(\tilde{W}, 2 \tilde{L}) \simeq H^{0}\left(X, 2 K_{X, D}\right)$. It follows that $\operatorname{Quad}\left(X, K_{X, D}\right)$ contains the image of $\tilde{W}$ under the morphism induced by $|\tilde{L}|$. $(\tilde{W}, \tilde{L})$ gives us a 
weak Del Pezzo surface whose homogeneous ideal is known to be generated by quadrics. Therefore $\operatorname{Quad}\left(X, K_{X, D}\right)$ is the weak Del Pezzo surface of degree $g-2$.

Lemma 3.5. Assume that $X$ is a canonical curve of genus 9 which has a simple $g_{8}^{3}$. Then $\operatorname{Quad}\left(X, K_{X, D}\right)$ is a weak Del Pezzo surface of degree seven.

Proof. Let $M$ be the line bundle on $X$ giving the $g_{8}^{3}$. Then it is normally generated because it attains the Castelnuovo's upper bound. Since $\operatorname{Sym}^{2} H^{0}(X, M) \rightarrow H^{0}(X, 2 M)=H^{0}\left(X, K_{X}\right)$ has one-dimensional kernel, the image $\Phi_{M}(X)$ lies on a quadric surface. It is either non-singular or a cone over the conic curve. In the latter case, we blow $\mathbb{P}^{3}$ up at the vertex to get a non-singular surface isomorphic to $\Sigma_{2}$. Therefore, we can embedd $X$ as a hypersurface of a geometrically ruled surface $W$ linearly equivalent to $4 H$, where $H$ denote the pull-back to $W$ of a hyperplane of $\mathbb{P}^{3},\left.H\right|_{X}=M$. Let $\mu: \tilde{W} \rightarrow W$ be the blowing-up at $D \in X$, and put $E=\mu^{-1}(D)$. We identify $X$ with the proper transform by $\mu$ in $\tilde{W}$. Then $K_{X, D}$ is induced by $2 \mu^{*} H-E$. We easily see that the restriction gives us isomorphisms $H^{0}\left(\tilde{W}, 2 \mu^{*} H-E\right) \simeq H^{0}\left(X, K_{X, D}\right)$ and $H^{0}\left(\tilde{W}, 4 \mu^{*} H-2 E\right) \simeq H^{0}\left(X, 2 K_{X, D}\right)$. This shows that $\operatorname{Quad}\left(X, K_{X, D}\right)$ is the image of $\tilde{W}$ by the morphism induced by $\left[2 \mu^{*} H-E\right]$ which is a weak Del Pezzo surface of degree 7.

We remark that $X$ is either bi-elliptic or has a $g_{6}^{2}$ when $g=6,7$.

\section{$\S 3.2$. General case}

In what follows, we assume that our $X$ has a $g_{4}^{1}$ and that $g \geq 8$. We frequently use the following notation. Let $\Sigma_{d}$ be the Hirzebruch surface of degree $d \geq 0$, that is, a $\mathbb{P}^{1}$-bundle $\mathbb{P}(\mathcal{O}(d) \oplus \mathcal{O})$ on $\mathbb{P}^{1}$. We denote by $\Delta_{0}$ the minimal section (that is, a section with self-intersection $-d$ ) and by $\Gamma$ a fiber.

Let $f: X \rightarrow \mathbb{P}^{1}$ be the morphism of degree 4 . Let $\mathcal{E}$ be the saturated subsheaf of $f_{*} \mathcal{O}_{X}\left(K_{X, D}\right)$ generated by $H^{0}\left(\mathbb{P}^{1}, f_{*} \mathcal{O}_{X}\left(K_{X, D}\right)\right)$. Then we have $\operatorname{rk}(\mathcal{E})=3, h^{0}\left(\mathbb{P}^{1}, \mathcal{E}\right)=g-1$ and $\operatorname{deg}(\mathcal{E})=g-4$. The natural sheaf homomorphism $f^{*} \mathcal{E} \hookrightarrow f^{*} f_{*} \mathcal{O}_{X}\left(K_{X, D}\right) \rightarrow \mathcal{O}_{X}\left(K_{X, D}\right)$ induces an embedding $\psi: X \rightarrow W=\mathbb{P}(\mathcal{E})$ over $\mathbb{P}^{1}$. We identify $X$ with $\psi(X)$. We let $H$ be the tautological line bundle and $\Gamma$ a fiber of the projection $\pi: \mathbb{P}(\mathcal{E}) \rightarrow \mathbb{P}^{1}$. Then $\left.H\right|_{X}=K_{X, D}$

Since the multiplication map $\operatorname{Sym}^{2} H^{0}\left(X, K_{X, D}\right) \rightarrow H^{0}\left(X, 2 K_{X, D}\right)$ is surjective and it factors through $H^{0}(W, 2 H)$, we see that the restriction map 
$H^{0}(W, 2 H) \rightarrow H^{0}\left(X, 2 K_{X, D}\right)$ is also surjective. Let $\mathcal{I}_{X / W}$ denote the ideal sheaf of $X$ in $W$ and consider the exact sequence

$$
0 \rightarrow \mathcal{I}_{X / W}(2 H) \rightarrow \mathcal{O}_{W}(2 H) \rightarrow \mathcal{O}_{X}\left(2 K_{X, D}\right) \rightarrow 0
$$

We have $H^{1}\left(W, \mathcal{I}_{X / W}(2 H)\right)=0$. Hence, we get $H^{0}\left(R^{1} \pi_{*} \mathcal{I}_{X / W}(2 H)\right)=0$ and $H^{1}\left(\pi_{*} \mathcal{I}_{X / W}(2 H)\right)=0$ by the Leray spectral sequence. Then $R^{1} \pi_{*} \mathcal{I}_{X / W}(2 H)=$ 0 , essentially because the restriction map $H^{0}\left(\mathbb{P}^{2}, \mathcal{O}(2)\right) \rightarrow H^{0}\left(Z, \mathcal{O}_{Z}(2)\right)$ is surjective for a generic 0 -cycle $Z$ of length 4 , implying that $R^{1} \pi_{*} \mathcal{I}_{X / W}(2 H)$ is at most a torsion sheaf. Hence taking direct images, we get the exact sequence

$$
0 \rightarrow \pi_{*} \mathcal{I}_{X / W}(2 H) \rightarrow \operatorname{Sym}^{2}(\mathcal{E}) \rightarrow f_{*} \mathcal{O}_{X}\left(2 K_{X, D}\right) \rightarrow 0
$$

By the Riemann-Roch theorem and the Leray spectral sequence, we can compute the rank and the degree of $f_{*} \mathcal{O}_{X}\left(2 K_{X, D}\right)$ to see $\operatorname{rk}\left(f_{*} \mathcal{O}_{X}\left(2 K_{X, D}\right)\right)=4$ and $\operatorname{deg}\left(f_{*} \mathcal{O}_{X}\left(2 K_{X, D}\right)\right)=3 g-9$. Since $\operatorname{rk}\left(\operatorname{Sym}^{2} \mathcal{E}\right)=6$ and $\operatorname{deg} \operatorname{Sym}^{2}(\mathcal{E})=$ $4(g-4)$, we get $\operatorname{rk}\left(\pi_{*} \mathcal{I}_{X / W}(2 H)\right)=2$ and $\operatorname{deg}\left(\pi_{*} \mathcal{I}_{X / W}(2 H)\right)=g-7$. From $h^{1}\left(\mathbb{P}^{1}, \pi_{*} \mathcal{I}_{X / W}(2 H)\right)=0$, it follows $h^{0}\left(\mathbb{P}^{1}, \pi_{*} \mathcal{I}_{X / W}(2 H)\right)=g-5$. Therefore, if we write $\pi_{*} \mathcal{I}_{X / W}(2 H)=\mathcal{O}_{\mathbb{P}^{1}}(\alpha) \oplus \mathcal{O}_{\mathbb{P}^{1}}(\beta)$ with two integers $\alpha, \beta(\alpha \geq \beta)$, we get $\beta \geq-1$ and $\alpha+\beta=g-7$.

Note that the natural inclusion $\mathcal{O}(\alpha) \hookrightarrow \mathrm{Sym}^{2} \mathcal{E}$ gives us an inclusion $\mathcal{O} \hookrightarrow \operatorname{Sym}^{2} \mathcal{E}(-\alpha)$. Hence $1 \in H^{0}\left(\mathbb{P}^{1}, \mathcal{O}\right)$ induces a section of $\operatorname{Sym}^{2} \mathcal{E}(-\alpha)$. Through the identification $H^{0}\left(\operatorname{Sym}^{2} \mathcal{E}(-\alpha)\right) \simeq H^{0}(W, 2 H-\alpha \Gamma)$, it defines a relative hyperquadric $Q_{1}$ which contains $X$ by the construction. Similarly, the inclusion $\mathcal{O}(\beta) \hookrightarrow \mathrm{Sym}^{2} \mathcal{E}$ gives us an relative hyperquadric $Q_{2}$ containing $X$, $Q_{2} \in|2 H-\beta \Gamma|$.

Assume first that $\beta \geq 0$. Then $\left|2 H-Q_{2}\right| \neq \emptyset$ and we have $X \subset Q_{1} \cap Q_{2}$. We see that $\operatorname{Quad}\left(X, K_{X, D}\right)$ is the image of $Q_{1} \cap Q_{2}$ under the morphism induced by $|H|$. We can compute the degree of $Q_{1} \cap Q_{2}$ as

$$
(2 H-\alpha \Gamma)(2 H-\beta \Gamma) H=4 H^{3}-2(\alpha+\beta) H^{2} \Gamma=4(g-4)-2(g-7)=2 g-2
$$

On the other hand, $\operatorname{deg} X=\operatorname{deg} K_{X, D}=2 g-3$. Therefore, $Q_{1} \cap Q_{2}$ contains a line. It is nothing but the trisecant line of $X$ detected in Lemma 2.6.

If $\beta=-1$, then $\left|2 H-Q_{2}\right|=\emptyset$ and $\alpha=g-6$. Hence the image of $Q_{1}$ under the morphism induced by $|H|$ is nothing $\operatorname{but} \operatorname{Quad}\left(X, K_{X, D}\right)$. It is easy to see that it is a weak Del Pezzo surface because its degree in $\mathbb{P} H^{0}\left(X, K_{X, D}\right)$ is given by $(2 H-(g-6) \Gamma) H^{2}=2(g-4)-(g-6)=g-2$. We shall show with several lemmas that $X$ is either a bi-elliptic curve or has a $g_{6}^{2}$ or a $g_{8}^{3}$.

Put $\mathcal{E}=\mathcal{O}(a) \oplus \mathcal{O}(b) \oplus \mathcal{O}(c)$ with integers $a, b, c$ satisfying $a \geq b \geq c \geq 0$ and $a+b+c=g-4$. We can take sections $Z_{0}, Z_{1}$ and $Z_{2}$ of $[H-a \Gamma],[H-b \Gamma]$ and 
$[H-c \Gamma]$, respectively, such that they form a system of homogeneous coordinates on any fiber of $\pi$. Then the equation defining $Q_{1} \in|2 H-(g-6) \Gamma|$ can be written as

$$
\sum_{i+j=2,0 \leq i, j \leq 2} \varphi_{i j} Z_{0}^{i} Z_{1}^{j} Z_{2}^{2-i-j}
$$

where the $\varphi_{i j}$ 's are forms of degree $i a+j b+(2-i-j) c-(g-6)$ on the base curve $\mathbb{P}^{1}$. If $2 b<g-6$, then it can be divided by $Z_{0}$, which is absurd. Hence $2 b \geq g-6$. Then the possible types of $(a, b, c)$ are as follows:

- $g=2 n+2(n \geq 3),(a, b, c)=(n-2, n-2,2),(n-1, n-2,1),(n-1, n-$ $1,0),(n, n-2,0)$

- $g=2 n+1(n \geq 4),(a, b, c)=(n-2, n-2,1),(n-1, n-2,0)$

Lemma 3.6. For $n \geq 5$, the following cases are impossible: $(a, b, c)=$ $(n-2, n-2,2),(n-1, n-2,1),(n-2, n-2,1)$.

Proof. The equation of $Q_{1}$ is of the form

$$
\varphi_{1} Z_{0}^{2}+\varphi_{2} Z_{0} Z_{1}+\varphi_{3} Z_{1}^{2}=0
$$

If $(a, b, c)=(n-2, n-2,2)$, then the $\varphi_{i}$ 's are constant. It follows that $Q_{1}$ is reducible, which is absurd. Assume that $(a, b, c)=(n-1, n-2,1)$ or $(n-$ $2, n-2,1)$. Then $Q_{1}$ is singular along $Z_{0}=Z_{1}=0$. Let $\mu: \tilde{W} \rightarrow W$ be the blow-up with center $\left\{Z_{0}=Z_{1}=0\right\}$. Then $\tilde{W}$ is isomorphic to the total space of the $\mathbb{P}^{1}$-bundle

$$
\varpi: \mathbb{P}\left(\mathcal{O}_{V}(\Gamma) \oplus \mathcal{O}_{V}\left(H_{1}\right)\right) \rightarrow V=\mathbb{P}(\mathcal{O}(a) \oplus \mathcal{O}(b)) \simeq \Sigma_{a-b}
$$

where $H_{1}$ denotes a tautological divisor on $V$. Since the exceptional divisor for $\mu$ is linearly equivalent to $\mu^{*} H-\varpi^{*} H_{1}$, we see that the proper transform $\tilde{Q}_{1}$ of $Q_{1}$ is linearly equivalent to $\mu^{*}(2 H-(g-6) \Gamma)-2\left(\mu^{*} H-\varpi^{*} H_{1}\right)=$ $\varpi^{*}\left(2 H_{1}-(g-6) \Gamma\right)$. This implies that $\tilde{Q}_{1}$ is isomorphic to a $\mathbb{P}^{1}$-bundle over a curve $C \in\left|2 H_{1}-(g-6) \Gamma\right|$. Note that $C$ is of arithmetic genus zero. Since $Q_{2}$ is a relative hyperquadric, we see that $X$ is a double covering of the rational curve $C$, which is absurd because we have assumed that $\operatorname{Cliff}(X)=2$.

Lemma 3.7. If $g=10$, then the cases $(a, b, c)=(2,2,2),(3,2,1)$ are impossible.

Proof. Assume that $(a, b, c)=(2,2,2)$. Then $\mathbb{P}(\mathcal{E}) \simeq \mathbb{P}^{2} \times \mathbb{P}^{1}$. Through this identification, for the irreducible relative hyperquadric $Q_{1} \in|2 H-4 \Gamma|$, 
there is a non-singular conic curve $C$ such that $Q_{1} \simeq C \times \mathbb{P}^{1}$. Hence $X$ can be regarded as a divisor on $\Sigma_{0}$. But it can be easily shown that a non-singular curve of genus 10 on $\Sigma_{0}$ has either a $g_{2}^{1}$ or a $g_{3}^{1}$. This is impossible when $\operatorname{Cliff}(X)=2$.

We next assume that $(a, b, c)=(3,2,1)$. Then the equation of $Q_{1}$ is of the form

$$
\varphi_{1} Z_{0}^{2}+\varphi_{2} Z_{0} Z_{1}+\varphi_{3} Z_{1}^{2}+\varphi_{4} Z_{0} Z_{2}=0
$$

where $\operatorname{deg} \varphi_{1}=2, \operatorname{deg} \varphi_{2}=1$ and $\varphi_{3}$ and $\varphi_{4}$ are constants. If $\varphi_{3}=0$, then the equation can be divided by $Z_{0}$, which is absurd. If $\varphi_{4}=0$, we get a contradiction as in the previous lemma. Hence $\varphi_{3}$ and $\varphi_{4}$ are non-zero constants. Then by a change of the coordinates, we can assume that $Q_{1}$ is defined by $Z_{0} Z_{2}-Z_{1}^{2}=0$. Then the projection map $\left.\pi\right|_{Q_{1}}: Q_{1} \rightarrow \mathbb{P}^{1}$ gives $Q_{1}$ a $\mathbb{P}^{1}$-bundle structure. Since $Z_{0} Z_{1} Q_{1}=(H-3 \Gamma)(H-2 \Gamma)(2 H-4 \Gamma)=-2$, the section $\left\{Z_{0}=Z_{1}=0\right\}$ induces the minimal section with self-intersection number -2 . Hence $Q_{1}$ is isomorphic to $\Sigma_{2}$. But, it is easy to see that a non-singular curve of genus 10 on $\Sigma_{2}$ has either a $g_{2}^{1}$ or a $g_{3}^{1}$.

Lemma 3.8. If $g=9$ and $(a, b, c)=(2,2,1)$, then $X$ has either $a g_{6}^{2}$ or $a g_{8}^{3}$.

Proof. The equation of $Q_{1} \in|2 H-3 \Gamma|$ is of the form

$$
\varphi_{1} Z_{0}^{2}+\varphi_{2} Z_{0} Z_{1}+\varphi_{3} Z_{1}^{2}+\varphi_{4} Z_{0} Z_{2}+\varphi_{5} Z_{1} Z_{2}=0
$$

where $\operatorname{deg} \varphi_{1}=\operatorname{deg} \varphi_{2}=\operatorname{deg} \varphi_{3}=1$ and $\operatorname{deg} \varphi_{4}=\operatorname{deg} \varphi_{5}=0$. If $\varphi_{4}=$ $\varphi_{5}=0$, then we will get a contradiction as in the proof of Lemma 3.6. By a coordinate change among $Z_{0}$ and $Z_{1}$, we can assume that $\varphi_{4}=1$ and $\varphi_{5}=0$. Then, by a suitable change of coordinates, we can normalize the equation as $Z_{0} Z_{2}+\varphi Z_{1}^{2}=0$, where $\operatorname{deg} \varphi=1$. This shows that $Q_{1}$ is a non-singular surface and $\left.\pi\right|_{Q_{1}}: Q_{1} \rightarrow \mathbb{P}^{1}$ has only one singular fiber over the zero of $\varphi$. We have $Z_{0} Z_{1} Q_{1}=(H-2 \Gamma)^{2}(2 H-3 \Gamma)=-1$. Hence $Q_{1}$ is $\Sigma_{1}$ blown up at a point which is not on the minimal section. Let $\mu: Q_{1} \rightarrow \Sigma_{1}$ be the corresponding blowingup. We denote by $E$ the exceptional $(-1)$ curve and by $\bar{\Gamma}$ the proper transform of the fiber over $(\varphi)$. Assume that $X$ is linearly equivalent to $\mu^{*}\left(4 \Delta_{0}+x \Gamma\right)-y E$ on $Q_{1}$, where $x, y$ are non-negative integers with $x \geq 4$. Since $X \cdot \bar{\Gamma} \geq 0$, we have $y \leq 4$. Since $K_{X}$ is induced by $\mu^{*}\left(2 \Delta_{0}+(x-3) \Gamma\right)-(y-1) E$, we have $\left(\mu^{*}\left(4 \Delta_{0}+x \Gamma\right)-y E\right)\left(\mu^{*}\left(2 \Delta_{0}+(x-3) \Gamma\right)-(y-1) E\right)=16$. From this, we get $6 x=y(y-1)+36$ and see that the possible $(x, y)$ 's are

$$
(x, y)=(6,0),(6,1),(7,3),(8,4)
$$


In the first two cases, $\mu$ can map $X$ isomorphically to $\Sigma_{1}$ and $X$ is linearly equivalent to $4 \Delta_{0}+6 \Gamma$. Through the blow-down $\Sigma_{1} \rightarrow \mathbb{P}^{2}$ of the minimal section $\Delta_{0}$, we get a plane curve model of $X$ which is a sextic with a double point. In the last two cases of $(x, y)$, we can blow $\bar{\Gamma}$ down to a point to get $\Sigma_{0}$ on which $X$ is still non-singular and is linearly equivalent to $4 \Delta_{0}+4 \Gamma$. Therefore, $X$ has a simple $g_{8}^{3}$.

Lemma 3.9. Assume that $(a, b, c)$ is either $(n-1, n-1,0),(n-1, n-$ $2,0)$ with $n \geq 4$ or $(n, n-2,0)$ with $n \geq 3$. Then $X$ is a bi-elliptic curve and the image of $Q_{1}$ under the morphism induced by $H$ is a cone over a non-singular elliptic curve.

Proof. In these cases, $\Phi_{H}(\mathbb{P}(\mathcal{E}))$ is a cone over the surface $V=\mathbb{P}(\mathcal{O}(a) \oplus$ $\mathcal{O}(b)) \simeq \Sigma_{a-b}$ embedded by the tautological line bundle $H_{1}$. We consider the $\mathbb{P}^{1}$-bundle $\tilde{W}=\mathbb{P}\left(\mathcal{O} \oplus \mathcal{O}\left(H_{1}\right)\right)$ over $V$ obtained as the proper transform of the cone via the blowing-up of $\mathbb{P} H^{0}\left(X, K_{X, D}\right)$ at the vertex. Then we have a surjective morphism $\mu: \tilde{W} \rightarrow W$ which contracts the inverse image of the vertex to the section of $W$ given by $Z_{0}=Z_{1}=0$.

We first assume that $Q_{1}$ is singular along $Z_{0}=Z_{1}=0$. Note that this is the case unless $(a, b, c)=(4,2,0),(3,2,0)$. Since $Q_{1}$ is singular along $Z_{0}=Z_{1}=0$, the proper transform $\tilde{Q}_{1}$ in $\tilde{W}$ is linearly equivalent to $\left(2 \mu^{*} H-(g-6) \Gamma\right)-$ $2\left(\mu^{*} H-\varpi^{*} H_{1}\right)=\varpi^{*}\left(2 H_{1}-(g-6) \Gamma\right)$, where $\varpi: \tilde{W} \rightarrow V$ denotes the natural projection map. This implies that $\tilde{Q}_{1}$ is a $\mathbb{P}^{1}$-bundle over a curve $C$ defined in $V$ by a section of $\left[2 H_{1}-(g-6) \Gamma\right]$. It is easy to see that $C$ is of arithmetic genus one. The proper transform of $X$ by $\mu$ is in $\tilde{Q}_{1}$ and the natural projection $\tilde{Q}_{1} \rightarrow C$ induces on $X$ a double covering of $C$. By the assumption that $\operatorname{Cliff}(X)=2$, we conclude that $C$ must be a non-singular elliptic curve. Hence $X$ is a bi-elliptic curve and the image of $\tilde{Q}_{1}$ under the morphism induced by $\mu^{*} H$ is the cone over $C$ embedded by $\left.H_{1}\right|_{C}$, which is nothing but $\operatorname{Quad}\left(X, K_{X, D}\right)$.

We next assume that $Q_{1}$ is not singular along $Z_{0}=Z_{1}=0$. We only have to consider the two cases $(a, b, c)=(4,2,0),(3,2,0)$. In these cases, the equation of $Q_{1}$ is of the form

$$
\varphi_{1} Z_{0}^{2}+\varphi_{2} Z_{0} Z_{1}+\varphi_{3} Z_{1}^{2}+c Z_{0} Z_{2}=0
$$

with a non-zero constant $c$ (because $Q_{1}$ is not singular along $Z_{0}=Z_{1}=0$ ). Hence, by a suitable change of coordinates, it can be transformed into a canonical form

$$
Z_{0} Z_{2}+\varphi Z_{1}^{2}=0
$$


where $\operatorname{deg} \varphi=1$. Then, as in the proof of Lemma 3.8, we see that $Q_{1}$ is $\Sigma_{4}$ (resp. $\left.\Sigma_{5}\right)$ blown up at a point $\notin \Delta_{0}$ when $(a, b, c)=(4,2,0)$ (resp. $(a, b, c)=$ $(3,2,0))$. In both cases, $X$ is a non-singular curve in $Q_{1}$ of respective genus 10,9 , and the natural projection $Q_{1} \rightarrow \mathbb{P}^{1}$ gives $X$ a 4 -to- 1 map. Using this description, one can immediately check that such $X$ cannot exist as in the proof of Lemma 3.8.

Lemma 3.10. Assume that $g=8$ and $(a, b, c)=(2,1,1),(2,2,0)$. Then $X$ is bi-elliptic or has a $g_{6}^{2}$.

Proof. Assume that $(a, b, c)=(2,1,1)$. Then the equation of $Q_{1}$ is of the form

$$
\varphi_{1} Z_{0}^{2}+\varphi_{2} Z_{0} Z_{1}+\varphi_{3} Z_{0} Z_{2}+c_{1} Z_{1}^{2}+c_{2} Z_{1} Z_{2}+c_{3} Z_{2}^{2}=0
$$

where $\operatorname{deg} \varphi_{1}=2, \operatorname{deg} \varphi_{2}=\operatorname{deg} \varphi_{3}=1$ and the $c_{i}^{\prime} s$ are constants. Since $Q_{1}$ is irreducible, we have $\left(c_{1}, c_{2}, c_{3}\right) \neq(0,0,0)$. We consider the morphism of $W$ induced by $|H-\Gamma|$ and we denote it by $\nu: W \rightarrow \mathbb{P}^{3}$. Note that $\nu$ is defined by $\left(X_{0}: X_{1}: X_{2}: X_{3}\right)=\left(t_{0} Z_{0}: t_{1} Z_{0}: Z_{1}: Z_{2}\right)$ and that it is considered as the blowing-up of $\mathbb{P}^{3}$ with center $\ell=\left\{X_{0}=X_{1}=0\right\}$. If $Q$ denotes the image of $Q_{1}$ by $\nu$, then it is a quadric surface which does not contain $\ell$. Since $\left.[H-\Gamma]\right|_{X}=K_{X, D}-F$ is of degree $9, \nu$ induces a birational morphism of $X$ onto its image $Y$ because $X$ has no $g_{3}^{1}$. Recall that $Q_{1}$ is obtained from $Q$ by blowing up $\ell \cap Q$ and $X$ is the proper transform in $Q_{1}$ of $Y$. It follows that $Y$ has at most two singular points (possibly infinitely near). We now calculate the arithmetic genus of $Y$. Assume that $Q \simeq \Sigma_{0}$ and that $Y$ is linearly equivalent to $\alpha \Delta_{0}+\beta \Gamma$ with $\alpha, \beta \geq 4$. Then, since $\operatorname{deg} Y=9$, we can assume that $\alpha=4$ and $\beta=5$, giving that $Y$ is of arithmetic genus 12 . Since $X$ is of genus 8 and $Y$ has at most two singular points, we conclude that $Y$ has a triple point $P$. Then the projection from $P$ induces on $X$ a $g_{6}^{2}$. Assume that $Q$ is a cone over a conic curve. Taking the resolution, we can assume that $Y$ is on $\Sigma_{2}$. Then it is easy to see that $Y$ is linearly equivalent to $4 \Delta_{0}+9 \Gamma$. So, $Y$ is of arithmetic genus 12 and, as in the previous case, we conclude that $X$ has a $g_{6}^{2}$.

We next assume that $(a, b, c)=(2,2,0)$. Then the equation of $Q_{1}$ is

$$
\varphi_{1} Z_{0}^{2}+\varphi_{2} Z_{0} Z_{1}+\varphi_{3} Z_{1}^{2}+c_{1} Z_{0} Z_{2}+c_{2} Z_{1} Z_{2}=0
$$

where $\operatorname{deg} \varphi_{1}=\operatorname{deg} \varphi_{2}=\operatorname{deg} \varphi_{3}=2$ and the $c_{i}$ 's are constants. If $c_{1}=c_{2}=0$, then we can show that $X$ is a bi-elliptic curve as in the proof of Lemma 3.9. Hence we can assume that $c_{1} \neq 0, c_{2}=0$ by a suitable linear change of coordinates among $Z_{0}, Z_{1}$. Then putting $\varphi_{1} Z_{0}+\varphi_{2} Z_{1}+c_{1} Z_{2}$ to be new $Z_{2}$, 
the equation can be transformed to

$$
Z_{0} Z_{2}+\varphi_{3} Z_{1}^{2}=0
$$

We have $Z_{0} Z_{1}(2 H-2 \Gamma)=-2$. Therefore, if $\varphi_{3}=0$ has two different roots, $Q_{1}$ is $\Sigma_{2}$ blown up two distinct points $P_{1}, P_{2}$ which are on different fibers and not on the minimal section. Let $\mu: Q_{1} \rightarrow \Sigma_{2}$ be the blow-up and $E_{i}=\mu^{-1}\left(P_{i}\right)$. We assume that $X$ is linearly equivalent to $\mu^{*}\left(4 \Delta_{0}+x \Gamma\right)-y_{1} E_{1}-y_{2} E_{2}$. Note that we have $x \geq 8,0 \leq y_{1}, y_{2} \leq 4$. We may further assume that $y_{1} \geq y_{2}$. Since $X$ is of genus 8 , we have $6 x=y_{1}\left(y_{1}-1\right)+y_{2}\left(y_{2}-1\right)+46$. It follows that $\left(x, y_{1}, y_{2}\right)=(8,2,0),(8,2,1),(9,3,2),(10,4,2)$. Therefore $\mu(X)$ is a curve of degree $x$ with a singular point $P_{1}$ of multiplicity $y_{1}=x-6$, and we see that $X$ has a $g_{6}^{2}$ by considering the projection of $\mu(X)$ with center $P_{1}$. If $\varphi_{3}=0$ has a multiple root, then $Q_{1}$ has an ordinary double point (that is, a singularity of type $A_{1}$ ) and the minimal resolution $\hat{Q}_{1}$ of $Q_{1}$ is $\Sigma_{2}$ blown up two points which are infinitely near and not on $\Delta_{0}$. Also in this case we can show that $X$ has a $g_{6}^{2}$ by considering the proper transform of $X$ in $\hat{Q}_{1}$ similarly as in the previous case.

In summary, we have shown the following:

Theorem 3.1. Assume that $\operatorname{Cliff}(X)=2, g \geq 6$ and $\operatorname{deg} D=1$. Then $X=\Phi_{X, D}(X)$ has a trisecant line $\ell$ and

$$
\operatorname{Quad}\left(X, K_{X, D}\right)=X \cup \ell
$$

unless, either

(1) $X$ is bi-elliptic, or

(2) $X$ has a simple $g_{6}^{2}$ (in particular $g \leq 10$ ), or

(3) $g=9$ and $X$ has a simple $g_{8}^{3}$.

In these exceptional cases, $\operatorname{Quad}\left(X, K_{X, D}\right)$ is a weak Del Pezzo surface of degree $g-2$.

\section{Acknowledgement}

I am very grateful to Professor Shigeru Mukai who informed me of his interesting result several years ago. I also thank my teacher, Professor Makoto Namba, for his constant encouragement for years. Apparently, my interests in low dimensional algebraic varieties originate from his beautiful works on curves. 


\section{References}

[1] Arbarello, E., Cornalba, M., Griffiths, P. A. and Harris, J., Geometry of Algebraic Curves, Volume I, GMW 267, Springer-Verlag, 1985.

[2] Coppens, M. and Martens, G., Secant spaces and Clifford's theorem, Compositio Math., 78 (1991), 193-212.

[3] Eisenbud, D., Lange, H., Martens, G. and Schreyer, F.-O., The Clifford dimension of a projective curve, Compositio Math., 72 (1989), 173-204.

[4] Green, M., Koszul cohomology and geometry of projective varieties, J. Diff. Geom., 19 (1984), 125-171.

[5] Green, M. and Lazarsfeld, R., On the projective normality of linear series on an algebraic curve, Invent. Math., 83 (1986), 73-90.

[6] Fujita, T., On the structure of polarized varieties with $\Delta$-genera zero, J. Fac. Sci. Univ. of Tokyo, 22 (1975), 103-115.

[7] Lange, H. and Sernesi, E., Quadrics containing a Prym-canonical curve, J. Alg. Geom., 5 (1996), 387-399.

[8] Namba, M., Familes of Meromorphic Functions on Compact Riemann Surfaces, Lect. Notes in Math., 767, Springer-Verlag, Berlin, 1979. 